Departamento de Educación Médica, Facultad de Medicina, Universidad de Concepción. Concepción, Chile. angeniero Informático, Magíster en Educación Médica para las Ciencias de la Salud.

bEnfermera, Magíster en Envejecimiento y Calidad de Vida.

Fuente de apoyo Financiero: Fondecyt Regular № 1170525

Recibido el 1 de marzo de 2018, aceptado el 29 de junio de 2018.

Correspondencia a: Liliana Ortiz-Moreira lilianaortiz@udec.cl

\section{Educación médica y diversidad cultural en Chile. Un diálogo pendiente}

\author{
LILIANA E. ORTIZ-MOREIRA, EDUARDO A. FASCE-HENRY, \\ OLGA B. MATUS-BETANCOURT ${ }^{\mathrm{a}}$, IVONE CAMPOS C. ${ }^{\mathrm{b}}$
}

\section{Medical education and cultural diversity in Chile. A pending dialogue}

\begin{abstract}
Medical education in Chile has a good research productivity. National educators are skilled in complex curricular design processes and in didactic innovation. However, the question of what it means to be doctor in a society that moves towards interculturality, has not been addressed thoroughly. Using the structure of a clinical record, we outline our critical view about the relationship between medical education and diversity. To describe the medical history, we refer to some variables of the training process such as the Hegemonic Model of Medicine, the epistemological beliefs of the students, the hidden curriculum and the educational environment. Then, the empirical evidence of the clinical picture, diagnosis and internationally recommended treatment is described, to end with a local therapeutic proposal.
\end{abstract}

(Rev Med Chile 2018; 146: 922-928)

Key words: Cultural Diversity: Education, Medical; Models, Educational.

\section{L} a universidad es el espacio social que permite generar conocimiento, transformar al ser y pensar la sociedad que queremos. Entre sus funciones más reconocidas se cuenta la de formar profesionales socialmente responsables. Para lograr su cometido es necesario un vínculo estrecho con la población, que permita identificar sus necesidades con el propósito de actualizar planes de estudio, estrategias de enseñanza, entre otros. Esta tarea no siempre es simple ni oportuna, dado que demanda una comprensión de la realidad social, que en el caso de la medicina chilena prioriza aspectos biomédicos ${ }^{1}$. Por lo anterior, pese a los avances científicos y tecnológicos, aún experimentamos lo que Erich Fromm denominó "sociedad enferma" 2 . Entre sus síntomas y signos se puede reconocer a través de la historia la vulneración de los derechos de los grupos minoritarios de la sociedad, entre ellos a las mujeres, los pueblos originarios, las minorías sexuales (LGBT) y las personas en situación de discapacidad ${ }^{3}$. Chile sigue siendo una sociedad altamente desigual en cuestión de ingreso, riqueza, salud y educación a pesar del fuerte crecimiento económico alcanzado en las últimas décadas ${ }^{4}$. Y esta desigualdad impacta el acceso a las prestaciones de salud y los indicadores sanitarios de grupos vulnerables ${ }^{5-8}, y$ posiblemente también las interacciones y prácticas profesionales en universidades y campos clínicos.

En este artículo exponemos una reflexión, desde la educación médica, que permita a los gestores curriculares y académicos de las escuelas de medicina tomar "conciencia de enfermedad", con el propósito de contribuir al mejoramiento del "diagnóstico" (identificación de necesidades sociales) y "plan terapéutico"(rediseños curriculares), en un momento histórico en el que nuestro país aumenta la diversidad y multiculturidad como consecuencia, entre otros factores, de la migración de ciudadanos y médicos extranjeros ${ }^{9}$, $\mathrm{y}$ del reconocimiento de grupos minoritarios que presentan demandas sanitarias inéditas o poco familiares para la medicina que aprendemos $y$ enseñamos $^{3,10}$. 


\section{Anamnesis próxima}

El motivo de consulta es que los educadores médicos y estudiantes de medicina, en la práctica clínica replican conductas que históricamente han sido aceptadas, manteniendo estereotipos a nivel de las interacciones y del discurso con el equipo de salud, el paciente $y / o$ su familia ${ }^{11}$. El prejuicio inconsciente de los médicos, como resultado de privilegios sociales no reconocidos contribuye a estas disparidades en salud ${ }^{12}$.

Aunque en Chile se ha estudiado poco este fenómeno, los efectos son visibles. En un estudio realizado por un equipo de la Universidad de Chile, se apreció que la discriminación dificulta a las mujeres lesbianas o bisexuales recibir una atención digna, oportuna, igualitaria y desprejuiciada en el área ginecológica, mientras que el terreno de la salud mental algunos psiquiatras o psicólogos siguen considerando a las orientaciones sexuales como un problema, lo cual deriva en diagnósticos, tratamientos o recomendaciones erradas ${ }^{3}$. De igual manera, y en el caso específico de los homosexuales, el principal problema detectado fue la asociación automática entre orientación sexual y VIH/SIDA, lo cual se traduce en que los médicos consideren siempre a una persona como sospechosa de vivir con el virus única y exclusivamente por ser homosexual ${ }^{3}$.

En cuanto a la diversidad al interior del gremio, aunque no existen datos oficiales, se observa aumento de las postulantes y egresadas de medicina. Esto puede apoyarse en las cifras oficiales de ingreso a las instituciones de educación superior en el año 2016, que contó con 51,6\% de mujeres matriculadas que eligen de preferencia carreras del área de la Salud, Ciencias Sociales, y Educación. También crece el número de mujeres en especialidades quirúrgicas. Asimismo, un porcentaje significativo de los educadores médicos son mujeres. Si bien ha disminuido la brecha de género, los cargos de responsabilidad y por ende la toma de decisiones en las universidades corresponden mayoritariamente a los hombres.

\section{Anamnesis remota}

Entre los antecedentes mórbidos se pueden describir algunos fenómenos o variables que contribuyen a la reproducción de estereotipos en medicina:

\section{Modelo médico hegemónico}

La educación médica en Chile está impregnada de rasgos estructurales propios del llamado "modelo médico hegemónico" (MMH) ${ }^{1}$. Este modelo (medicina facultativa u ortodoxa), se entiende como "un conjunto de prácticas, saberes y teorías generadas por el desarrollo de la medicina científica, que desde su origen desplazó los modelos que dominaban en la sociedad, hasta lograr identificarse como la única forma de entender y atender la enfermedad $^{13}$. El MMH ha sido legitimado tanto por criterios científicos como por el Estado, encontrando un antecedente filosófico en la dicotomía cuerpo-mente propuesta por Descartes que ha marcado la investigación científica y la praxis médica en el mundo occidental ${ }^{1}$. El informe Flexner, elaborado en el año 1910 para la transformación de la Educación Médica en USA y Canadá, es uno de los factores que contribuyó a la legitimación del $\mathrm{MMH}^{14}$. También se reconoce la influencia del curriculum oculto, del clima educacional y de los modelos profesionales ${ }^{15}$. No obstante, de la definición de Menéndez se desprende que el MMH es jerárquico, asimétrico, clasista, racista y mercantil. Además, Sala advierte que el reduccionismo médico derivado de la excesiva división disciplinar y fragmentación del conocimiento constituye un verdadero problema para la salud pública, propiciando actitudes potencialmente peligrosas para los diversos integrantes de los sistemas de salud ${ }^{16}$.

\section{Proceso de enseñanza}

Durante los primeros años de la formación, el estudiante es inducido a explorar la mayor cantidad de explicaciones posibles y puede equivocarse sin que ello tenga repercusiones en otro; desde quinto año, sus reflexiones deben ser rápidas y traducirse en decisiones concretas y correctas. Esto último, por economía cognitiva, inseguridad y pragmatismo podría automatizarse a expensas de habilidades cognitivas de baja complejidad ${ }^{17}$. En otras palabras, el proceso de enseñanza transforma las creencias de los estudiantes sobre la naturaleza del conocimiento (epistemología), desde procesos sofisticados de tipo constructivista hacia el reduccionismo propio del $\mathrm{MMH}$.

Otras variables que puedan dar cuenta de esta transformación son aspectos disciplinarios, actitudinales, motivacionales, clima educacional y modelos profesionales a los que están expuestos los estudiantes ${ }^{17}$. 


\section{Clima educacional}

Aunque la versión del instrumento utilizado para comparar el clima de seis escuelas de medicina chilenas no incluía ítems que midieran la percepción de discriminación, los resultados permiten un correlato indirecto con el MMH y las creencias de los estudiantes. La dimensión peor evaluada fue percepción social lo que se atribuye, entre otros, a que los docentes se perciben más autoritarios, sin capacidad de estimular la auto-confianza. Se encontró una variabilidad significativa entre escuelas, aunque éstas no fueron identificadas ${ }^{18}$.

\section{Antecedentes empíricos}

De la enfermedad: existen dudas sobre si la educación médica prepara adecuadamente a los médicos para atender a las personas de grupos minoritarios ${ }^{19}$. Las políticas de no discriminación y la capacitación existente parecen no mejorar las actitudes y el conocimiento de los médicos en esta materia ${ }^{20}$. Los propios estudiantes de medicina reconocen brechas de conocimiento para abordar los problemas relacionados con la cultura que surgen en la atención del paciente ${ }^{21}$. Pese a lo anterior, una proporción sustancial de estudiantes no creen que la autorreflexión de los propios sesgos culturales sea importante para el desempeño como médicos; ni consideran importante la comprensión de las diferentes creencias culturales de los pacientes como parte la atención efectiva ${ }^{21}$.

Por otro lado, la diversidad racial y étnica en la profesión, como estrategia en USA para enfrentar la crisis en la disparidad de atención recibida por grupos minoritarios, está siendo afectada por la prohibición de la acción afirmativa en los procesos de selección de estudiantes ${ }^{22}$. De hecho, en el estudio de Dhaliwal, encontró que solo $37 \%$ de los estudiantes cree que la escuela es diversa ${ }^{23}$.

En relación con el clima educacional, en este mismo estudio los resultados son preocupantes: aunque la mayor parte de los participantes considera que el clima es amigable con grupos minoritarios, muchos informaron que han visto a otros estudiantes, residentes y profesores hacer comentarios despectivos o mostrar comportamientos ofensivos hacia personas de estos grupos ${ }^{23}$. En otro estudio, los estudiantes de medicina también reportaron observar discriminación por sexo o género, pero ellos autopercibían un alto nivel de comodidad y confianza brindando atención médica a pacientes $\mathrm{LGBT}^{24}$. Además, otro estudio reportó la asociación entre mayor exposición a un clima educacional negativo de diversidad y el aumento en los síntomas depresivos autoinformados por estudiantes de medicina ${ }^{25}$.

Asimismo, la cultura del entorno laboral inhibe el éxito profesional de las mujeres en la academia $^{26}$. Sumado a lo anterior, la falta de modelos para combinar las responsabilidades familiares y profesionales, las frustraciones con las tareas de investigación, el equilibrio trabajo-vida son factores claves asociado con la decisión de abandonar la academia ${ }^{27}$. Eso se traduce en baja representación de las mujeres en cargos de decanos, intervalos de tiempo más largos para ser promovidas a profesor titular, y menor tiempo de permanencia en sus cargos que sus contrapartes ${ }^{28}$.

Del diagnóstico: Se ha demostrado que la equidad de género tiene un impacto positivo en las actividades cotidianas de las personas (toma de decisiones, asignación de ingresos, aplicación y cumplimiento de normas / reglas) que afectan su salud. La equidad de género también es un determinante crucial de las desigualdades en salud; por lo tanto, el monitoreo es importante para la vigilancia de la salud de mujeres y hombres, así como para las futuras iniciativas de políticas de salud $^{5}$. Además, los estudiantes enfrentarán un aumento en la población de pacientes LGBT mayores o en proceso de envejecimiento, con necesidades sanitarias únicas ${ }^{10}$. En ese mismo sentido, Nesbit recomienda prestar atención a los factores culturales y sociales que determinan disparidad para mejorar los resultados en salud ${ }^{29}$.

\section{Del tratamiento: se describen organizadas por niveles de responsabilidad}

\section{Planificación estratégica}

En el año 2012, Nivet divulgó tres fases implementadas por la Association of American Medical Colleges para la evolución del pensamiento de la diversidad en educación médica. La primera incluyó esfuerzos aislados encaminados a eliminar barreras sociales y legales al acceso y la igualdad, considerando la excelencia institucional y la diversidad como fines competitivos. La segunda fase mantuvo la diversidad en la periferia, pero generó 
conciencia sobre los beneficios de ésta, permitiendo que la excelencia y la diversidad existan como fines paralelos. La tercera fase refleja la comprensión de la relevancia de la diversidad extendida a las instituciones y los sistemas: diversidad e inclusión se integran en el funcionamiento central de la institución y se enmarcan como estándares para lograr la excelencia ${ }^{30}$. En el año 2015, el mismo autor identificó cinco nuevas:

- Definiciones más amplias de diversidad para incluir a las personas LGBT y las personas con discapacidades;

- Cargos administrativos importantes en la escuela de medicina para líderes en diversidad;

- Uso creciente de un enfoque holístico para evaluar a los postulantes a la carrera;

- Reconocimiento de la diversidad y la inclusión como un estándar de excelencia de las escuelas de medicina;

- Apreciación de la importancia de las subpoblaciones dentro de grupos minoritarios e insuficientemente representados ${ }^{31}$.

\section{Perfil de egreso y diseño curricular}

La adquisición de habilidades para reconocer y corregir los determinantes sociales adversos de la enfermedad es un componente importante de la educación médica de pregrado ${ }^{12,32,33}$. Los médicos y otros trabajadores de la salud están llamados a cerrar las brechas culturales que pueden existir entre ellos y sus pacientes. Una educación intercultural adecuada es esencial para reducir las disparidades existentes en la atención de la salud ${ }^{21}$.

\section{Proceso de enseñanza}

La enseñanza de la justicia social para corregir las desigualdades en salud requiere aprendizaje autodirigido, autorreflexión, crecimiento personal y didáctica centrada en el estudiante ${ }^{12,34,35}$. Una propuesta sencilla, pero útil se basó en el uso de vivencias de los profesores para crear un "espacio seguro" y facilitar discusiones en las que los alumnos pudieran desarrollar una conciencia crítica. Durante las interacciones reconocieron y abordaron explícitamente cuestiones relacionadas con las diferencias de poder, el racismo, el prejuicio y el sesgo de género ${ }^{36}$.

\section{Clima educacional}

Se reportaron cambios positivos en las percepciones del clima de diversidad a lo largo del tiem- po: en la práctica de la medicina; en el currículo, incluido el contenido del curso; en las clases y en la interacción docente-alumno. También se informó una disminución significativa en la percepción de sesgo de género experimentado o presenciado, aunque los eventos informados solo mostraron pequeñas disminuciones. Los autores consideran que estos resultados pueden explicarse por una aceptación generacional de la diversidad cultural y por la inclusión de cursos de diversidad en el plan de estudios de medicina ${ }^{37}$.

\section{Plan terapéutico}

Citando a Nivet, el avance hacia un sistema de salud más equitativo requiere una transformación de cómo financiamos, ofrecemos y evaluamos la atención médica. Pero también requiere un nuevo rol de la diversidad e inclusión como una solución a nuestros problemas, en vez de seguir considerándolo como un problema que se debe solucionar ${ }^{31}$.

Las políticas sanitarias chilenas han avanzado hacia la equidad. Paralelamente, el Ministerio de Educación ha hecho esfuerzos por mejorar el acceso a la educación superior de personas afectadas por injusticias sociales. También se ha logrado avanzar en el financiamiento de la educación superior. Sin embargo, aún estamos lejos de curar a nuestra "sociedad enferma". Esto se refleja en el acuerdo $\mathrm{N}^{\circ}$ 025/2018 de la Comisión Nacional de Educación, que elimina la formación en filosofía y ciencias en enseñanza media, áreas fundamentales en el desarrollo del pensamiento crítico, lógico y científico, que a su vez constituyen la base para alcanzar los principios de equidad y no discriminación.

Pero no todo está perdido. En relación a la gestión del rediseño e innovación curricular en medicina, los educadores médicos chilenos hemos ampliado nuestra sensibilidad y competencias para esta compleja tarea. En la actualidad, somos capaces de darnos cuenta que la construcción del currículum supone un tipo de conocimiento, que debe ser concebido como un objeto dinámico y no lineal, en interacción permanente con sus diversas fuentes y actores; y que su construcción demanda un modelo participativo y transparente que garantice la validez y la legitimidad de la propuesta formativa, mediante la reflexión y la negociación de sentido entre los diversos participantes ${ }^{38}$. 
En ese mismo sentido, desde 2017, tres escuelas de medicina chilena iniciaron un proyecto que busca analizar el posible efecto que las concepciones acerca de diversidad e inclusión, tanto de docentes como de estudiantes de carreras de la salud, pueden tener sobre sus experiencias académicas. Los resultados permitirán contribuir a fomentar la inclusión a nivel de la sociedad a través de una mejor experiencia académica de docentes y estudiantes que forman parte de la sociedad chilena, en la que estas temáticas se están volviendo cada vez más relevantes.

El objetivo de esta revisión es cuestionar el Status Quo, invitarlos a salir de su zona de confort, reflexionar de manera crítica y abordar la realidad mediante un pensamiento sistémico ${ }^{39}$ que permita acelerar nuestro tránsito por las fases necesarias para que la diversidad y la inclusión sean fines y estándares de calidad de las escuelas de medicina chilenas.

\section{Referencias}

1. Outomuro Delia. Fundamentación de la enseñanza de la Bioética en Medicina. Acta bioeth. [Internet]. 2008; 14 (1): 19-29. Disponible en: https:// scielo.conicyt.cl/scielo.php?script=sci_arttext\&pi$\mathrm{d}=$ S1726-569X2008000100003\&lng=es. http://dx.doi. org/10.4067/S1726-569X2008000100003 [citado el 1 de marzo de 2018].

2. Goodman B. Erich Fromm: a sane society? Mental health in an insane world. Nurse Educ Today 2013; 33 (3): 185-6. doi: 10.1016/j.nedt.2012.09.011. Epub 2012 Oct 9. PubMed PMID: 23062867.

3. Montero A, Gonzáez MI. Guía de orientaciones y recomendaciones para la atención de adolescentes y jóvenes gays, lesbianas y bisexuales en el nivel primario de salud [Internet] 2016. Disponible en: http://www. medicina.uchile.cl/documentos/orientaciones-y-recomendaciones-para-la-atencion-de-adolescentes-y-jovenes-gays-lesbianas-y-bisexuales-en-el-nivel-primario-de-salud_124057_0_2206.pdf [citado el 9 de mayo de 2018].

4. OCDE (2018). Estudios económicos de la OCDE, Chile. Febrero 2018.

5. Fernández-Sáez J, Ruiz-Cantero MT, Guijarro-Garví M, Carrasco-Portiño M, Roca-Pérez V, Chilet-Rosell E, et al. Looking twice at the gender equity index for public health impact. BMC Public Health 2013; 13: 659. doi: 10.1186/1471-2458-13-659. PubMed PMID: 23855520; PubMed Central PMCID: PMC3751633.
6. Espinoza MA, Cabieses B. Equidad en Salud y Evaluación de Tecnologías Sanitarias en Chile. Rev Med Chile 2014; 142 (Suppl 1): 45-9. Disponible en: https:// scielo.conicyt.cl/scielo.php?script=sci_arttext\&pi$\mathrm{d}=$ S0034-98872014001300008\&lng=es. http://dx.doi. org/10.4067/S0034-98872014001300008 [citado el 28 de febrero de 2018].

7. Fasce E, Fasce F, Zárate H, Campos I, Flores M, Ibáñez P. Relación entre perímetro abdominal, nivel socioeconómico y presión arterial. Rev Chil Cardiol [Internet]. 2010; 29 (1): 11-8. Disponible en: https:// scielo.conicyt.cl/scielo.php?script $=$ sci_arttext\&pi$\mathrm{d}=$ S0718-85602010000100001\&lng=es. http://dx.doi. org/10.4067/S0718-85602010000100001 [citado el 1 de marzo de 2018].

8. Teixidó-Compañó E, Espelt A, Sordo L, Bravo MJ, Sarasa-Renedo A, Indave BI, et al. Differences between men and women in substance use: the role of educational level and employment status. Gac Sanit 2018; 32 (1): 41-7. doi: 10.1016/j.gaceta.2016.12.017. Epub 2017 Mar 18. PubMedPMID: 28318754.

9. Lazzaro-Salazar M, Pujol-Cols LJ. Riesgos psicosociales e integración profesional de médicos extranjeros: Un estudio sobre el manejo del conflicto en Chile. Rev Med Chile [Internet]. 2017 ; 145 (10): 1300-7. Disponible en: https://scielo.conicyt.cl/scielo.php?script=sci_arttext\&pid=S0034-98872017001001300\&lng=es. http:// dx.doi.org/10.4067/S0034-98872017001001300 [citado el 1 de marzo de 2018].

10. Cannon SM, Shukla V, Vanderbilt AA. Addressing the healthcare needs of older Lesbian, Gay, Bisexual, and Transgender patients in medical school curricula: a call to action. Med Educ Online 2017; 22 (1): 1320933. doi: 10.1080/10872981.2017.1320933. PubMed PMID: 28468575; PubMed Central PMCID: PMC5419296.

11. Franco M, Villarreal E, Vargas ER, Martínez L, Galicia L. Estereotipos negativos de la vejez en personal de salud de un Hospital de la Ciudad de Querétaro, México. Rev Med Chile [Internet]. 2010 ; 138 (8): 988-93. Disponible en: https://scielo.conicyt.cl/scielo.php?script=sci_arttext\&pid=S0034-98872010000800007\&lng=es. http:// dx.doi.org/10.4067/S0034-98872010000800007 [citado el 28 de febrero de 2018].

12. Holm AL, Rowe Gorosh M, Brady M, White-Perkins D. Recognizing Privilege and Bias: An Interactive Exercise to Expand Health Care Providers' Personal Awareness. Acad Med 2017; 92 (3): 360-4. doi: 10.1097/ ACM.0000000000001290. PubMed PMID: 27355785.

13. Menéndez EL. Modelo Médico Hegemónico y Atención Primaria. Segundas Jornadas de Atención Primaria de la Salud. Buenos Aires. 1988; 451-64. [Internet]. Dispo- 
nible en: http://www.psi.uba.ar/academica/carrerasdegrado/psicologia/sitios_catedras/electivas/816_rol_psicologo/material/unidad2/obligatoria/modelo_medico. pdf [citado el 28 de febrero de 2018].

14. Ortiz L. El legado flexneriano en el siglo 21. Resumen Bibliográfico. Rev Educ Cienc Salud 2010; 7 (1): 36-8.

15. Ortega J, Fasce E, Pérez C, Ibáñez P, Márquez C, Parra P. Evaluación de componentes del currículum oculto en estudiantes de medicina. Rev Med Chile [Internet]. 2014; 142 (11): 1452-7. Disponible en: https:// scielo.conicyt.cl/scielo.php?script=sci_arttext\&pi$\mathrm{d}=$ S0034-98872014001100013\&lng=es. http://dx.doi. org/10.4067/S0034-98872014001100013 [citado el 1 de marzo de 2018].

16. Sala JE. [The jibarization of logos: how medical reductionism can kill]. Bol Med Hosp Infant Mex 2017; 74 (2): 154-63. doi: 10.1016/j.bmhimx.2016.11.009. Epub 2017 Mar 17. Spanish. PubMed PMID: 29382499.

17. Lazcano X, Villalón F, Vera S, Conget P. La creencia epistemológica constructivista sobre el conocimiento científico varía en función del año de formación en los estudiantes de Medicina pero no en los estudiantes de otras carreras de la salud. Rev Med Chile [Internet]. 2017; 145 (9): 1184-92. Disponible en: https:// scielo.conicyt.cl/scielo.php?script=sci_arttext\&pi$\mathrm{d}=$ S0034-98872017000901184\&lng=es. http://dx.doi. org/10.4067/s0034-98872017000901184 [citado el 1 de marzo de 2018].

18. Herrera C, Pacheco J, Rosso F, Cisterna C, Aichele D, Becker S, et al. Evaluación del ambiente educacional pre-clínico en seis Escuelas de Medicina en Chile. Rev Med Chile [Internet]. 2010; 138 (6): 677-84. Disponible en: https://scielo.conicyt.cl/scielo.php?script=sci_arttext\&pid=S0034-98872010000600003\&lng=es. http:// dx.doi.org/10.4067/S0034-98872010000600003 [citado el 1 de marzo de 2018].

19. Wear D, Zarconi J, Aultman JM, Chyatte MR, Kumagai AK. Remembering Freddie Gray: Medical Education for Social Justice. Acad Med 2017; 92 (3): 312-7. doi: 10.1097/ACM.0000000000001355. PubMed PMID: 27580436.

20. Jabson JM, Mitchell JW, Doty SB. Associations between non-discrimination and training policies and physicians' attitudes and knowledge about sexual and gender minority patients: a comparison of physicians from two hospitals. BMC Public Health 2016; 16: 256. doi: 10.1186/s12889-016-2927-y. PubMed PMID: 26968373; PubMed Central PMCID: PMC4788836.

21. Garces LM, Mickey-Pabello D. Racial Diversity in the Medical Profession: The Impact of Affirmative Action Bans on Underrepresented Student of Color Matricu- lation in Medical Schools. J Higher Educ 2015; 86 (2): 264-94. PubMed PMID: 26052161; PubMed Central PMCID: PMC4454423.

22. Loue S, Wilson-Delfosse A, Limbach K. Identifying Gaps in the Cultural Competence/Sensitivity Components of an Undergraduate Medical School Curriculum: A Needs Assessment. J Immigr Minor Health 17 (5): 1412-9, 2015 Oct.

23. Dhaliwal JS, Crane LA, Valley MA, Lowenstein SR. Student perspectives on the diversity climate at a U.S. medical school: the need for a broader definition of diversity. BMC Res Notes. 2013; 6: 154. doi: 10.1186/1756-05006-154. PubMed PMID: 23595029; PubMed Central PMCID: PMC3684552.

24. Nama N, MacPherson P, Sampson M, McMillan HJ. Medical students' perception of lesbian, gay, bisexual, and transgender (LGBT) discrimination in their learning environment and their self-reported comfort level for caring for LGBT patients: a survey study. Med Educ Online 2017; 22 (1): 1368850. doi: 10.1080/10872981.2017.1368850. PubMed PMID: 28853327; PubMed Central PMCID: PMC5653936.

25. Hardeman RR, Przedworski JM, Burke S, Burgess DJ, Perry S, Phelan S, et al. Association Between Perceived Medical School Diversity Climate and Change in Depressive Symptoms Among Medical Students: A Report from the Medical Student CHANGE Study. J Natl Med Assoc 2016; 108 (4): 225-35. doi: 10.1016/j. jnma.2016.08.005. Epub 2016 Sep 24. PubMed PMID: 27979008 ; PubMed Central PMCID: PMC5171227.

26. Friede A, Speck R, Dupuis M. A Culture Conducive to Women's Academic Success: Development of a Measure. Academic Medicine 2012; 87 (11): 1622-31.

27. Levine R, Lin F, Kern D, Wright S, Carrese J. Stories From Early-Career Women Physicians Who Have Left Academic Medicine: A Qualitative Study at a Single Institution. Academic Medicine 2011; 86: 752-8.

28. White S, McDade S, Yamagata E, Morahan P. Gender-Related Differences in the Pathway to and Characteristics of U.S. Medical School Deanships. Academic Medicine 2012; 87 (8): 1-9.

29. Nesbitt S, Palomarez RE. Review: Increasing Awareness and Education on Health Disparities for Health Care Providers. Ethn Dis 2016; 26 (2): 181-90. doi: 10.18865/ ed.26.2.181.

30. Nivet MA. Commentary: Diversity 3.0: a necessary systems upgrade. Acad Med 2011; 86 (12): 1487-9. doi: 10.1097/ACM.0b013e3182351f79. PubMed PMID: 22130259.

31. Nivet MA. A Diversity 3.0 Update: Are We Moving the Needle Enough? Acad Med 2015; 90 (12): 1591-3. doi: 
10.1097/ACM.0000000000000950. PubMed PMID: 26422594.

32. Coria A, McKelvey TG, Charlton P, Woodworth M, Lahey T. The design of a medical school social justice curriculum. Acad Med 2013; 88 (10): 1442-9. doi: 10.1097/ACM.0b013e3182a325be. PubMed PMID: 23969356.

33. Bourgois P, Holmes SM, Sue K, Quesada J. Structural Vulnerability: Operationalizing the Concept to Address Health Disparities in Clinical Care. Acad Med 2017; 92 (3): 299-307. doi: 10.1097/ACM.0000000000001294. PubMed PMID: 27415443; PubMed Central PMCID: PMC5233668.

34. Ambrose AJ, Andaya JM, Yamada S, Maskarinec GG. Social justice in medical education: strengths and challenges of a student-driven social justice curriculum. Hawaii J Med Public Health 2014; 73 (8): 244-50. PubMed PMID: 25157325; PubMed Central PMCID: PMC4142578.

35. Schiff T, Rieth K. Projects in medical education: "Social Justice in Medicine" a rationale for an elective program as part of the medical education curriculum at John A. Burns School of Medicine. Hawaii J Med Public Health 2012; 71 (4 Suppl 1): 64-7. Review. PubMed PMID:
22737646; PubMed Central PMCID: PMC3347740.

36. Zaidi Z, Vyas R, Verstegen D, Morahan P, Dornan T. Medical Education to Enhance Critical Consciousness: Facilitators' Experiences. Acad Med 2017; 92 (11S Association of American Medical Colleges Learn Serve Lead: Proceedings of the 56th Annual Research in Medical Education Sessions):S93-S99. doi: 10.1097/ ACM.0000000000001907. PubMed PMID: 29065029.

37. Mujawar I, Sabatino M, Ray Mitchell S, Walker B, Weissinger P, Plankey M. A 12-year comparison of students' perspectives on diversity at a Jesuit Medical School. Med Educ Online 2014; 19: 23401. doi: 10.3402/meo. v19.23401. eCollection 2014.

38. Hawes G, Rojas-Serey AM, Espinoza M, Oyarzo S, Castillo-Parra S, Castillo M, et al. Desarrollo de una matriz conceptual para la innovación curricular en profesiones de la salud. Rev Med Chile [Internet]. 2017; 145 (9): 1193-7. Disponible en: https:// scielo.conicyt.cl/scielo.php?script $=$ sci_arttext\&pi$\mathrm{d}=$ S0034-98872017000901193\&lng=es. http://dx.doi. org/10.4067/s0034-98872017000901193 [citado el 1 de marzo de 2018].

39. Moran E. Los siete saberes para una educación del futuro. UNESCO, 1999. 